\title{
SUR LE RAPPORT ENTRE L'ACCROISSEMENT GOURANT EN DIAMETRE, EN HAUTEUR ET EN VOLUME DES ARBRES DANS LES PEUPLEMENTS VIERGES DE HETRE ORIENTAL (FAGUS ORIENTALIS Lipsky) EN IRAN*
}

\author{
par
}

\section{S.T. Nedialkov et A. Asli}

\section{0 . Introduction}

Les peuplements de hêtre oriental (Fagus orientalis Lipsky) en Iran sont répandus sur les versants nords du massif montagneux de l'Elbourz. En grande majorité, les peuplements sont vierges avec une structure multiple. En général, ces peuplements ont une production très élevée. Souvent on trouve des hêtres d'une hauteur allant jusqu'à $50 \mathrm{~m}$ et d'un diamètre à hauteur de poitrine de 150-180 cm. Dans la forêt de Sang Deh, par exemple, nous avons mesuré des peuplements avec un volume sur pied de $720 \mathrm{~m}^{3}$ par ha.

L'établissement de l'accroissement courant dans ces peuplements est très utile au point de vue de l'aménagement et de la sylviculture. Pour cela, il est nécessaire, bien sûr, d'utiliser des méthodes plus simples et plus justes. Afin d'établir les méthodes les plus convenables, il est nécessaire d'étudier les lois dans le rapport entre l'accroissement courant en diamètre, en hauteur et en volume. Il est certain que les études sur le sujet des peuplements vierges ne sont pas abondantes.

Les études que nous avons faites sont seulement une primairc dans ce domaine, c'est pourquoi nous sommes très loin de l'idée que nous allons résoudre tous les problèmes concernant l'accroissement courant des arbres dans les peuplements vierges de hêtre oriental, dans l'ouvrage présent.

\section{But et méthode des études}

Le but de l'étude présente est :

* Seminarie « Iran » - 12,12.1971. 
a) d'établir les lois dans les rapports entre l'accroissement courant en diamètre, en hauteur et en volume des arbres,

b) d'utiliser ces rapports afin d'établir plus justement l'accroissement courant en volume $-\hat{i}_{\mathrm{v}}$.

Dans ce but, on a analysé des tiges de trois arbres de différentes dimensions et âges. La caractéristique des arbres étudiés est présentée dans le tableau $\mathrm{n}^{\circ} 1$.

TABLEAU 1

\begin{tabular}{|c|c|c|c|}
\hline $\begin{array}{c}\text { Numéro } \\
\text { des arbres }\end{array}$ & $\begin{array}{c}\text { Age }(\mathrm{t}) \\
\text { ans }\end{array}$ & $\begin{array}{c}\text { Hauteur }(\mathrm{h}) \\
\mathrm{m} .\end{array}$ & $\begin{array}{c}\text { Diamètre à } 1,30 \mathrm{~m} . \\
\text { sur écorce }(\mathrm{d})-\mathrm{cm} .\end{array}$ \\
\hline 1 & 173 & 30,3 & 76,9 \\
2 & 140 & 29,7 & 40,8 \\
3 & 98 & 16,3 & 23,0 \\
\hline
\end{tabular}

Les arbres ont été choisis d'après l'âge ct le diamètre. On voit, d'après le tableau $n^{\circ} 1$ que les arbres $n^{\circ} 1$ et 2 sont âgés $(t)$, que leur diamètre à hauteur de poitrine est différent, mais que lès hauteurs (h) sont prèsque les mêmes.

L'arbre $n^{\circ} 3$ est très différent des arbres $n^{\circ} 1$ et $n^{\circ} 2$. Les tiges des arbres étudiés ont été analysées par période de 10 ans.

Afin d'établir les lois dans les rapports entre l'accroissement courant en diamètre, en hauteur et en volume des arbres, on a estimé, pour les différents âgés $(\mathrm{t})$, les éléments suivants :

$i_{h}$ - accroissement courant en hauteur (h) trine (d)

$i_{d}$ - accroissement courant en diamètre à hauteur de poi-

$\mathrm{Pi}_{\mathrm{yr}}$ - le pourcentage de l'accroissement réel en volume.

Les données établies pour $\mathrm{i}_{\mathrm{h}}, \mathrm{i}_{\mathrm{d}}$, et $\mathrm{Pi}_{\mathrm{vr}}$ sont présentées dans le tableau $\mathrm{n}^{\circ} 2$.

Afin d'établir la possibilité d'utiliser le rapport entre $i_{h}$, $i_{d}$, et $i_{v}$ pour l'estimation de $i_{v}$, on a dû utiliser les formules suivantes de Breiman :

$$
\mathrm{i}_{\mathrm{vi}}^{\prime}=\mathrm{v}\left(\frac{2 \mathrm{i}_{\mathrm{d}}}{\mathrm{d}}+\frac{\mathrm{i}_{\mathrm{h}}}{\mathrm{h}}\right)
$$

ou :

$i^{\prime}$ vi est l'accroissement courant en volume de tiges

$\mathrm{v}$ - volume actuel des tiges

$\mathrm{i}_{\mathrm{d}}$ - l'accroissement courant en diamètre

$\mathrm{d}$ - diamètre à hauteur de poitrine sans écorce

$i_{h}$ - l'accroissement courant en hauteur 
$\mathrm{h}$ - hauteur actuelle des tiges

$\mathrm{i}_{\mathrm{v} 2}^{\prime}=\mathrm{v} \frac{2 \mathrm{i}_{\mathrm{d}}}{\mathrm{d}}$

Dans la formule $\mathrm{n}^{\circ} 2$, les éléments sont évidents.

TABLEAU 2

\begin{tabular}{|c|c|c|c|c|c|c|c|c|c|}
\hline \multirow{2}{*}{$\begin{array}{c}\mathrm{t} \\
\text { ans }\end{array}$} & \multicolumn{3}{|c|}{ Arbre $N^{\circ} 1$} & \multicolumn{3}{|c|}{ Arbre No 2} & \multicolumn{3}{|c|}{ Arbre No 3} \\
\hline & $\begin{array}{c}\mathrm{i}_{\mathrm{h}} \\
\mathrm{cm} .\end{array}$ & $\begin{array}{c}\mathrm{i}_{\mathrm{d}} \\
\mathrm{mm}\end{array}$ & $\underset{\%}{\mathrm{Pi}_{\mathrm{yr}}}$ & $\begin{array}{l}\mathrm{i}_{\mathrm{h}} \\
\mathrm{cm} .\end{array}$ & $\begin{array}{c}\mathrm{i}_{\mathrm{d}} \\
\mathrm{mm}\end{array}$ & $\underset{\%}{\mathrm{Pi}_{\mathrm{yr}}}$ & $\begin{array}{l}\mathrm{i}_{\mathrm{h}} \\
\mathrm{cm} .\end{array}$ & $\begin{array}{c}\mathrm{i}_{\mathrm{d}} \\
\mathrm{mm} .\end{array}$ & $\underset{\%}{\mathrm{Pi}_{\mathrm{yr}}}$ \\
\hline 10 & 16 & 1,5 & 10,0 & 17 & 2,5 & 10,0 & 17 & 1,1 & 71 \\
\hline 20 & 16 & 40 & $\begin{array}{r}1,0 \\
8,5\end{array}$ & 26 & 3,5 & 9,6 & 28 & 1,9 & 5,1 \\
\hline 30 & 18 & 3,8 & 7,7 & 27 & 3,5 & 5,9 & 17 & 2,3 & 4,2 \\
\hline 40 & 22 & 4,0 & 6,3 & 18 & 2,6 & 4,9 & 20 & 2,2 & 3,1 \\
\hline 50 & 30 & 5,2 & 6,1 & 14 & 2,7 & 4,6 & 16 & 2,1 & 2,6 \\
\hline 60 & 26 & 5,1 & 5,6 & 22 & 2,8 & 3,9 & 10 & 2,7 & 1,9 \\
\hline 70 & 24 & 5,2 & 4,3 & 36 & 2,7 & 3,2 & 13 & 2,6 & 1,5 \\
\hline 80 & 17 & 4,5 & 3,4 & 28 & 2,0 & 3,0 & 13 & 3,8 & 1,0 \\
\hline 90 & 15 & 4,0 & 3,0 & 28 & 2,9 & 2,7 & 16 & 2,5 & 0,9 \\
\hline 98 & - & & & & & & 16 & 1,0 & 0,5 \\
\hline 100 & 13 & 4,3 & 2,8 & 21 & 3,1 & 2,0 & - & - & - \\
\hline 110 & 11 & 4,7 & 2,4 & 10 & 2,4 & 2,7 & - & - & - \\
\hline 120 & 15 & 4,4 & 2,1 & 18 & 3,2 & 3,7 & - & - & - \\
\hline 130 & 19 & 4,6 & 2,1 & 12 & 3,2 & 2,1 & - & - & - \\
\hline 140 & 16 & 4,2 & 2,1 & 10 & 2,7 & 1,9 & - & - & - \\
\hline 150 & 15 & 4,8 & 1,5 & - & 1 & 1 & - & - & - \\
\hline 160 & 14 & 4,6 & 1,5 & - & - & - & - & - & - \\
\hline 170 & 13 & 5,7 & 1,6 & - & - & - & - & - & - \\
\hline 173 & 10 & 4,3 & 1,2 & - & - & 一 & - & 一 & - \\
\hline
\end{tabular}

\section{Le rapport entre 1 'accroissement courant: $i_{h}, i_{d}$ et $P i_{v r}$}

On voit, dans le tableau $n^{\circ} 2$, que les changements de $i_{\text {th }}, i_{d}$ sont différents pour les trois arbres. L'arbre $n^{\circ} 1$ qui a été prèsque libre, sans concurrence des arbres de côté possède un accroissement courant en hauteur $\left(i_{h}\right)$ relativement faible, qui atteint le maximum de $30 \mathrm{~cm}$ à 50 ans.

L'arbre $\mathrm{n}^{\circ} 2$ qui a été très pressé par les arbres de côté possède un accroissement courant en hauteur $\left(i_{h}\right)$ relativement suffisant, l'arbre $n^{\circ} 2$ qui a 33 ans de moins que l'arbre $n^{\circ} 1$ a la même hauteur.

L'arbre $n^{\circ} 3$ qui est pressé du côté et d'en haut par les arbres voisins possède un accroissement en hauteur $\left(i_{h}\right)$ bien faible.

On voit, d'après le tableau $\mathrm{n}^{\circ} 2$ que l'accroissement courant en diamètre $\left(\mathbf{i}_{d}\right)$ dépend de la situation des arbres dans le peuplement. Le plus grand accroissement $\left(i_{d}\right)$ est celui de l'arbre $n^{\circ} 1$, 
suivi des arbres $n^{\circ} 2$ et $n^{\circ} 3$. L'arbre $n^{\circ} 1$, après 10 ans, a pendant toute la vie un accroissement courant en diamètre important, qui varie de $3,8 \mathrm{~mm}$ aे $5,7 \mathrm{~mm}$. On voit, en $173 \mathrm{ans}$, un accroissement de $4,3 \mathrm{~mm}$.

Les donnécs du tableau $n^{\circ} 2$ montrent que le pourcentage de l'accroissement réel en volume $\left(\mathrm{Pi}_{\mathrm{vr}}\right)$ diminue en fonction de l'augmentation de l'âge, dans tous les arbres.

Les valeurs les plus grandes de $\mathrm{Pi}_{\mathrm{vr}}$ sont dans les arbres $\mathrm{n}^{\circ} 1$ suivi des $\mathrm{n}^{\circ} 2$ et $\mathrm{n}^{\circ} 3$.

\section{Utilisation du rapport entre l'accroissement courant $i_{d}$ et $i_{h}$ pour l'estimation de l'accroissement en volume.}

L'accroissement courant réel en volume (ivr) a été estimé d'après la méthode d'analyse de tige. Ensuite, l'accroissement courant réel en volume $\left(i_{v r}\right)$ a été comparé avec l'accroissement courant en volume $i^{\prime}{ }_{v 1}$ calculé d'après la formule $n^{\circ} 1$ dans le tableau $\mathrm{n}^{\circ} 3$ et avec l'accroissement courant en volume $i_{\mathrm{v} 2}$ calculé d'après la formule $\mathrm{n}^{\circ} 2$ dans le tableau $\mathrm{n}^{\circ} 4$.

Les différences entre l'accroissement réel et l'accroissement calculé sont présentées en pourcentage dans les tableaux $n^{\circ} 3$ et $n^{\circ} 4$.

On voit d'après le tableau $\mathrm{n}^{\circ} 3$ que l'erreur dans l'estimation de l'accroissement par l'intermédiaire de la formule $\mathrm{n}^{\circ} 1$ en général, diminue en fonction de l'augmentation de l'âge des arbres. Cette formule donne une augmentation très importante de l'accroissement.

D'après le tableau $\mathrm{n}^{\circ} 4$, on voit que la formule $\mathrm{n}^{\circ} 2$ donne une diminution de l'accroissement. L'erreur de l'estimation de l'accroissement d'après cette formule est moins importante que l'erreur obtenue par l'intermédiaire de la formule $n^{\circ} 1$.

Dans la formule $\mathrm{n}^{\circ} 1$, on utilise pour l'estimation de l'accroissement le rapport entre $i_{v}, i_{d}$ et $i_{h}$. Il est certain que l'établissement de l'accroissement en hauteur $i_{h}$ des arbres sur pied est un travail très difficile. C'est pourquoi les résultats obtenus dans les tableaux $\mathrm{n}^{\circ} 3$ et $\mathrm{n}^{\circ} 4$ sont en faveur de la formule $\mathrm{n}^{\circ} 2$ dans laquelle on utilise seulement le rapport entre l'accroissement en volume ( $i_{v}$ ) et en diamètre $\left(i_{d}\right)$. Il est évident que l'accroissement courant en diamètre (id) s'estime très facilement.

Nous avons envisagé de présenter le rapport entre l'accroissement courant en volume ( $\left.i_{v}\right)$, en diamètre $\left(i_{d}\right)$ et en hauteur $\left(i_{h}\right)$ avec des coefficients empiriques. On a utilisé le coefficient 1,5, Les résultats obtenus sont présentés dans le tableau $n^{\circ} 5$. Dans ce tableau sont présentés les pourcentages des erreurs obtenues dans 
TABLEAU 3

\begin{tabular}{|c|c|c|c|c|c|c|c|c|c|}
\hline \multirow{2}{*}{$\begin{array}{c}t \\
\text { ans }\end{array}$} & \multicolumn{3}{|c|}{ Arbre $N^{0} 1$} & \multicolumn{3}{|c|}{ Arbre $N \circ 2$} & \multicolumn{3}{|c|}{ Arbre $N^{\circ} 3$} \\
\hline & $\begin{array}{l}\mathrm{i}_{\mathrm{h}} \\
\mathrm{cm} .\end{array}$ & $\begin{array}{c}\mathrm{i}_{\mathrm{d}} \\
\mathrm{mm} .\end{array}$ & $i_{v i}-i_{v r}$ & $\begin{array}{c}\mathrm{i}_{\mathrm{h}} \\
\mathrm{cm} .\end{array}$ & $\begin{array}{c}\mathrm{i}_{\mathrm{d}} \\
\mathrm{mm} .\end{array}$ & $i_{v i}-i_{v x}$ & $\begin{array}{c}i_{\text {h }} \\
\mathrm{cm} .\end{array}$ & $\begin{array}{c}\mathrm{i}_{\mathrm{d}} \\
\mathrm{mm} .\end{array}$ & $i_{\mathrm{vi}}-i_{\mathrm{vr}}$ \\
\hline 10 & 16 & 15 & +1200 & 17 & 25 & +2000 & 17 & 1 & 1500 \\
\hline $\begin{array}{l}10 \\
20\end{array}$ & $\begin{array}{l}10 \\
16\end{array}$ & $\begin{array}{l}1,3 \\
4,0\end{array}$ & $\begin{array}{l}+120,0 \\
+193,0\end{array}$ & 26 & $\begin{array}{l}2,5 \\
3,5\end{array}$ & $\begin{array}{r}+200,0 \\
+\quad 75,0\end{array}$ & 28 & $\begin{array}{l}1,1 \\
1,9\end{array}$ & $\begin{array}{r}+70,0 \\
+\quad 76,8\end{array}$ \\
\hline 30 & 18 & 3,8 & $\begin{array}{r}1 \\
+\quad 720\end{array}$ & 27 & 3,5 & $\begin{array}{r}10 \\
+\quad 854\end{array}$ & 17 & 2,3 & $\begin{array}{r}18,6 \\
+\quad 186\end{array}$ \\
\hline 40 & 22 & 4,0 & $\begin{array}{r}150 \\
+\quad 550\end{array}$ & 18 & 2,6 & $\begin{array}{r} \\
+\quad 26.4\end{array}$ & 20 & 22 & $\begin{array}{r}T \\
+\quad 167\end{array}$ \\
\hline 50 & 30 & 5,2 & $\begin{array}{r}+ \\
+\quad 48,2\end{array}$ & 14 & 2,7 & $\begin{array}{r}70,1 \\
+\quad 7,1\end{array}$ & 16 & 2,1 & $\begin{array}{r}1 \\
+\quad 848\end{array}$ \\
\hline 60 & 26 & 5,1 & $\begin{array}{r}10,4 \\
+\quad 314\end{array}$ & 22 & 2,8 & $\begin{array}{r}1 \\
+\quad 25.9\end{array}$ & 10 & 2,7 & $\begin{array}{r}1 \\
-\quad 08\end{array}$ \\
\hline 70 & 24 & 5,2 & $\begin{array}{r}+11 \\
+\quad 25,8\end{array}$ & 36 & 2,7 & $\begin{array}{r}+52,3 \\
+\end{array}$ & 13 & 2,6 & $\begin{array}{r}-5,8 \\
-\quad 5\end{array}$ \\
\hline 80 & 17 & 4,5 & $\begin{array}{r}13,8 \\
+\quad 138\end{array}$ & 28 & 2,0 & $\begin{array}{r}\quad 8,2 \\
+\quad 1\end{array}$ & 13 & 3,8 & $+22,1$ \\
\hline 90 & 15 & 4,0 & $+\quad 1,4$ & 28 & 2,9 & $\begin{array}{r}+31,1 \\
\end{array}$ & 16 & 2,5 & $\begin{array}{r}+8,8 \\
+\quad 8\end{array}$ \\
\hline 98 & - & - & - & & & - & 16 & 1,0 & $\begin{array}{r}7 \\
+\quad 79,2\end{array}$ \\
\hline 100 & 13 & 4,3 & $-1,2$ & 21 & 3,1 & $+\quad 2,3$ & - & - & - \\
\hline 110 & 11 & 4,7 & $+11,1$ & 10 & 2,4 & $-27,2$ & - & - & - \\
\hline 120 & 15 & 4,4 & $+17,2$ & 18 & 3,2 & $-30,6$ & - & - & - \\
\hline 130 & 19 & 4,6 & $+21,1$ & 12 & 3,2 & $\begin{array}{r}15 \\
+\quad 15\end{array}$ & - & - & - \\
\hline 140 & 16 & 4,2 & $-0,5$ & 10 & 2,7 & $-13,2$ & - & 一 & 一 \\
\hline 150 & 15 & 4,8 & $\begin{array}{r}31,7 \\
+\quad 4\end{array}$ & - & - & - & - & - & - \\
\hline 160 & 14 & 4,6 & $\begin{array}{r}126 \\
+226\end{array}$ & 一 & - & - & 一 & - & - \\
\hline 170 & 13 & 5,7 & $\begin{array}{r}137 \\
+\quad 24,7\end{array}$ & - & - & - & - & - & - \\
\hline 173 & 10 & 4,3 & $+\quad 1,1$ & - & - & - & - & - & - \\
\hline
\end{tabular}

TABLEAU 4

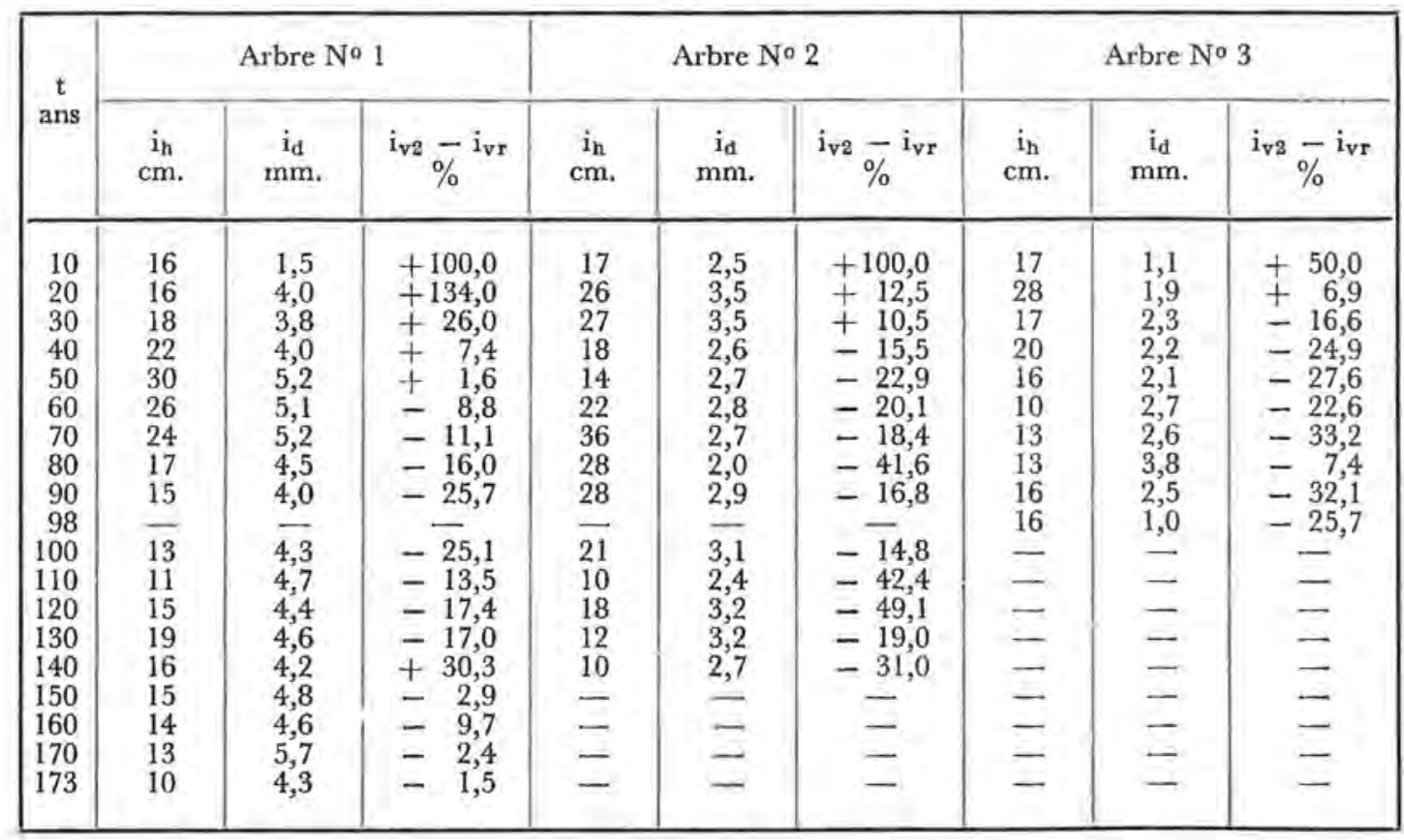


TABLEAU 5

\begin{tabular}{|c|c|c|c|c|c|c|c|c|c|c|c|c|}
\hline \multirow{2}{*}{$\begin{array}{c}\mathrm{t} \\
\text { ans }\end{array}$} & \multicolumn{4}{|c|}{ Arbre $N^{\circ} 1$} & \multicolumn{4}{|c|}{ Arbre $\mathrm{N}^{\circ} 2$} & \multicolumn{4}{|c|}{ Arbre No 3} \\
\hline & $\begin{array}{l}\mathrm{A} \\
\%\end{array}$ & $\begin{array}{l}\mathrm{B} \\
\%\end{array}$ & $\begin{array}{c}\mathrm{C} \\
\%\end{array}$ & $\underset{\%}{\mathrm{D}}$ & $\underset{\%}{\mathrm{~A}}$ & $\begin{array}{l}B \\
\%\end{array}$ & $\begin{array}{l}\mathrm{C} \\
\%\end{array}$ & $\begin{array}{l}\mathrm{D} \\
\%\end{array}$ & $\begin{array}{l}\mathrm{A} \\
\%\end{array}$ & $\begin{array}{c}\mathrm{B} \\
\%\end{array}$ & $\begin{array}{l}\mathrm{C} \\
\%\end{array}$ & $\begin{array}{l}\mathrm{D} \\
\%\end{array}$ \\
\hline $\begin{array}{r}10 \\
20 \\
30 \\
40 \\
50 \\
60 \\
70 \\
80 \\
90 \\
98 \\
100 \\
110 \\
120 \\
130 \\
140 \\
150 \\
160 \\
170 \\
173\end{array}$ & $\begin{array}{r}+120,0 \\
+193,0 \\
+72,0 \\
+55,0 \\
+48,2 \\
+31,4 \\
+25,8 \\
+13,8 \\
+\quad 1,4 \\
-1,2 \\
+\quad 11,1 \\
+17,2 \\
+21,1 \\
+\quad 0,5 \\
+41,7 \\
+22,6 \\
+24,7 \\
+\quad 1,1\end{array}$ & $\begin{array}{r}+50,0 \\
+107,0 \\
+\quad 41,0 \\
+22,7 \\
+23,2 \\
+\quad 8,6 \\
+\quad 3,6 \\
=\quad 7,1 \\
-17,2 \\
-\quad 20,1 \\
=13,3 \\
=4,1 \\
+\quad 0,4 \\
-17,9 \\
+\quad 14,1 \\
+\quad 0,1 \\
+\quad 0,4 \\
+\quad 2,1\end{array}$ & $\begin{array}{r}+100,0 \\
+134,0 \\
+26,0 \\
+\quad 7,4 \\
+\quad 1,6 \\
=8,8 \\
=11,1 \\
=16,0 \\
-26,7 \\
-25,1 \\
=13,5 \\
=17,4 \\
-17,0 \\
+\quad 30,3 \\
=\quad 2,9 \\
=\quad 9,7 \\
-\quad 2,4 \\
-\quad 1,5\end{array}$ & $\begin{array}{l}+50,0 \\
+\quad 76,0 \\
-\quad 5,5 \\
=19,6 \\
-24,9 \\
-31,6 \\
=33,3 \\
=37,0 \\
-44,3 \\
-43,3 \\
=35,1 \\
=36,2 \\
=37,8 \\
-47,7 \\
-22,9 \\
-32,2 \\
-26,8 \\
-26,1\end{array}$ & $\begin{array}{c}+200,0 \\
+75,0 \\
+85,4 \\
+26,4 \\
+7,1 \\
+25,9 \\
+52,3 \\
+8,2 \\
+31,1 \\
+-2,3 \\
-27,2 \\
-30,6 \\
+1,5 \\
-13,2 \\
= \\
=\end{array}$ & $\begin{array}{c}+150,0 \\
+47,7 \\
+55,5 \\
+\quad 5,2 \\
=12,1 \\
+6,2 \\
+31,8 \\
=6,4 \\
+10,3 \\
=1,0 \\
=41,5 \\
=43,3 \\
=18,8 \\
-30,2 \\
= \\
=\end{array}$ & $\begin{array}{r}+100,0 \\
+12,5 \\
+10,5 \\
-15,5 \\
=22,9 \\
=20,1 \\
=18,4 \\
=41,6 \\
-16,8 \\
=-14,8 \\
=42,4 \\
=49,1 \\
=19,0 \\
=-31,0 \\
=\end{array}$ & $\begin{array}{r}0,0 \\
-15,6 \\
-10,3 \\
-36,6 \\
=42,1 \\
=39,8 \\
=38,8 \\
=56,1 \\
-37,6 \\
-\overline{3}, 6,1 \\
=56,7 \\
=61,8 \\
=39,3 \\
-48,1 \\
=\end{array}$ & $\begin{array}{r}+200,0 \\
+112,5 \\
+46,9 \\
+41,3 \\
+32,9 \\
+25,0 \\
+16,5 \\
+52,9 \\
+54,4 \\
+103,8 \\
= \\
= \\
= \\
=\end{array}$ & $\begin{array}{r}+150,0 \\
+\quad 76,8 \\
+18,6 \\
+16,7 \\
+87,8 \\
+\quad 0,8 \\
-\quad 5,8 \\
+22,1 \\
+8,8 \\
+\quad 79,2 \\
= \\
= \\
= \\
= \\
=\end{array}$ & $\begin{array}{r}+100,0 \\
+\quad 4,4 \\
+\quad 10,9 \\
0,0 \\
-\quad 3,6 \\
+\quad 3,4 \\
-11,1 \\
+23,4 \\
=9,6 \\
=0,9 \\
= \\
= \\
= \\
= \\
=\end{array}$ & $\begin{array}{r}+50,0 \\
+\quad 6,9 \\
+\quad 16,6 \\
-24,9 \\
=27,6 \\
=22,6 \\
=33,2 \\
=7,4 \\
=32,1 \\
=25,7 \\
= \\
= \\
= \\
= \\
=\end{array}$ \\
\hline
\end{tabular}

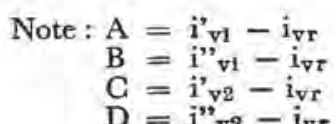


l'estimation d'après les formules $n^{\circ} 1$ et $n^{\circ} 2$ et les formules suivantes:

$$
\begin{aligned}
& i^{\prime \prime}{ }_{v 1}=v\left(\frac{1,5 i_{d}}{d}+\frac{i_{b}}{h}\right) \\
& i^{\prime \prime}{ }_{v 2}=v-\frac{1,5 i_{d}}{d}
\end{aligned}
$$

Les éléments, dans les formules $\mathrm{n}^{\circ} 3$ et $\mathrm{n}^{\circ} 4$, sont les mêmes que dans les formules $\mathrm{n}^{\circ} 1$ et $\mathrm{n}^{\circ} 2$, sauf le coefficient.

On voit, d'après le tableau $n^{\circ} 5$ que le pourcentage de l'erreur diminue si l'accroissement courant en volume est estimé avec lé coefficient 1,5 dans la formule $\mathrm{n}^{\circ} 3$ qui correspond à la formule $\mathrm{n}^{\circ} 1$.

Au contraire, le pourcentage de l'erreur augmente si l'accroissement courant en volume est estimé avec le coefficient 1,5 dans la formule $n^{\circ} 4$ qui correspond à la formule $n^{\circ} 2$.

\section{Gonclusion}

Les études effectuées sur le rapport entre l'accroissement courant en volume, en diamètre et en hauteur des arbres des peuplements multiples et vierges de hêttre oriental en Iran, montrent que la plus juste est la formule 3 pour l'estimation de l'accroissement courant en volume. Cette formule cependant est plus difficilement applicable parce qu'clle tient compte de l'accroissement en hauteur $\left(i_{\mathrm{h}}\right)$ pour l'établissement duquel on rencontre assez de difficultés pour les arbres sur pied.

C'est pourquoi la formule $n^{\circ} 2$ se montre plus convenable au point de vue pratique.

\section{SAMENVATTING}

Over het verband tussen de lopende aanwas en het stamvolume in natuurlijke bestanden van beuk in Iran.

De auteurs vergelijken verschillende formules, die het verband leggen tussen de lopende aanwas en enkele dendrometrische kenmerken van de boom.

$\mathrm{Zij}$ komen tot het besluit, dat de meest nauwkeurige resultaten worden bereikt met de formule

$$
\mathrm{i}_{\mathrm{v}}=\mathrm{v}\left(\frac{1,5 \mathrm{i}_{\mathrm{d}}}{\mathrm{d}}+\frac{\mathrm{i}_{\mathrm{h}}}{\mathrm{h}}\right)
$$

naar dat uit praktisch oogpunt de voorkeur moet gegeven worden aan de formule

$\mathrm{i}_{\mathrm{v}}=$ lopende volumeaanwas

$$
i_{\mathrm{v}}=\left(\mathrm{v} \frac{2 \mathrm{i}_{\mathrm{d}}}{\mathrm{d}}\right)
$$

$\mathrm{v}=$ actueel volume van de stam

$i_{d}=$ lopende diameteraanwas

$i_{\mathrm{h}}=$ lopende hoogtcaanwas

$\mathrm{d}=$ diameter op borsthoogte (zonder schors)

$\mathrm{h}=$ huidige stamlengte 


\section{RESUME}

Sur le rapport entre 1'accroissement courant en diamètre, en hauteur et en volume des arbres dans les peuplements vierges de hêtre oriental

Les auteurs font la comparaison de différentes formules pour établir le rapport entre l'accroissement courant et quelques caractéristiques dendrométrique de l'arbre.

Ils arrivent à la conclusion que le meilleur résultat est obtenu par l'application de la formule de base

$$
\dot{i_{v}}=v\left(\frac{1,5 i_{d}}{d}+\frac{i_{h}}{h}\right)
$$

mais que pour des raisons pratiques on préfère la formule suivante

$$
i_{v}=\left(v \frac{2 i_{d}}{d}\right)
$$

$\mathrm{i}_{\mathrm{v}}=$ accroissement courant

$\mathrm{v}=$ volume actuel des tiges

$\mathrm{i}_{\mathrm{d}}=$ accroissement courant en diamètre de la tige

h $=$ accroissement courant en hauteur de la tige

$\mathrm{d}=$ diamètre à hauteur de poitrine sans écorce

$\mathrm{h}=$ hauteur actuelle des tiges 